Vol. 84 No. 3 September 2019

\title{
CYTOLOGIA
}

International Journal of Cytogenetics and Cell Biology
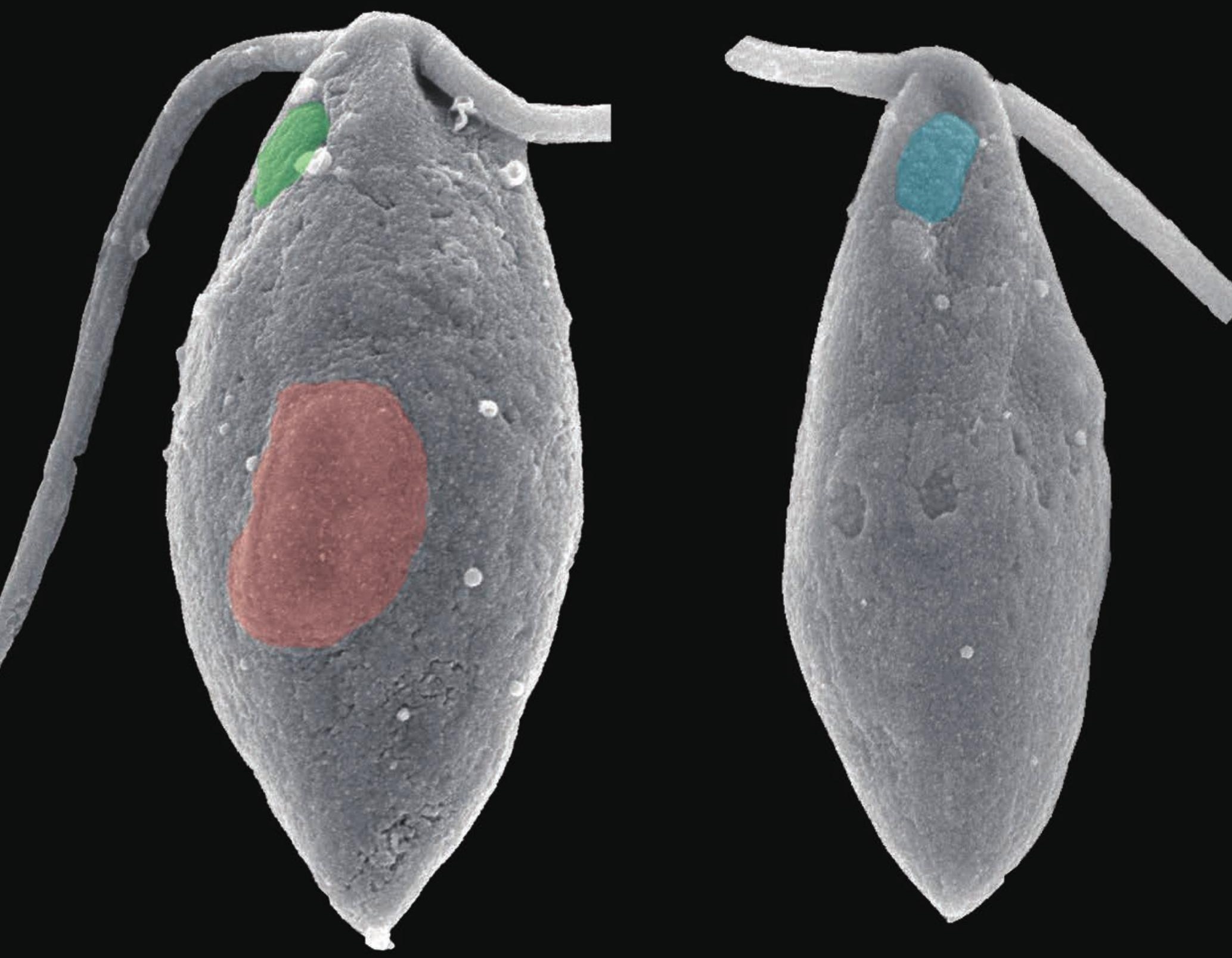


\title{
Technical Note:
}

\section{Visualization of Gamete Mating Structure of Marine Green Alga by FE-SEM}

\author{
Shinichi Miyamura ${ }^{1 *}$, Kensuke Ichihara $^{2}$, Tomokazu Yamazaki ${ }^{3}$, \\ Kazuyoshi Kuwano ${ }^{4}$ and Shigeyuki Kawano ${ }^{3}$
}

\footnotetext{
${ }^{1}$ Faculty of Life and Environmental Sciences, University of Tsukuba, 1-1-1 Tennodai, Tsukuba, Ibaraki 305-8572, Japan

${ }^{2}$ Field Science Center for Northern Biosphere, Hokkaido University, Funami-cho, Muroran, Hokkaido 051-0013, Japan

${ }^{3}$ Functional Biotechnology PJ, Future Center Initiative, The University of Tokyo, Wakashiba, Kashiwa, Chiba 277-0871, Japan

${ }^{4}$ Graduate School of Fisheries and Environmental Sciences, Nagasaki University, Bunkyo-machi, Nagasaki 852-8521, Japan
}

Eukaryotes generally have two sexes. Two sex/mating types are readily distinguishable based on the differences of gamete size and morphology in anisogamy and oogamy, while it is generally difficult to distinguish two sex/mating types in isogamy using such features (Miyamura et al. 2018). However, studies on chlorophyte algae have indicated that two gametes of the opposite mating types differ morphologically from each other irrespective of the gamete size difference. This feature is a sex specific positioning of the mating structure (specialized area of plasma membrane for gamete fusion) that was first found in Chlamydomonas reinhardtii. The mating structure of $\mathrm{mt}^{+}$gamete is located at the cell apex on the opposite side of the flagellar beat plane to the eyespot, whereas $\mathrm{mt}^{-}$structure is located on the same side as the eyespot. Field emission scanning electron microscopy (FE-SEM) has been used to identify the position of gamete mating structure in other species of chlorophyte algae and consequently sex specific positioning of the mating structure is found to be a widespread feature in the chlorophyte algae (Miyamura et al. 2015). However, it was still difficult to visualize the specialized area of plasma membrane such as mating structure by FE-SEM in most of the chlorophyte algae. To overcome this problem, we applied the osmium tetroxide-tannic acid-osmium tetroxide (OTO) method for processing samples to visualize the mating structure. This method reduces shrinkage of the sample during dehydration and preserves the plasma membrane structure.

Cover figure showed the FE-SEM image of female and male gamete of marine green alga Ulva arasakii (Ulvophyceae, Chlorophyta) prepared by OTO method. Cells were fixed with $2 \%$ glutaraldehyde comprising 3\% $\mathrm{NaCl}$ in $0.05 \mathrm{M}$ cacodylate buffer, $\mathrm{pH} 7.1$, on a Nuclepore polycarbonate membrane (Whatman Japan KK,
Tokyo, Japan) that was coated with $0.1 \%$ poly L-lysine. The cells were incubated at $4^{\circ} \mathrm{C}$ overnight and then washed in a series of $0.05 \mathrm{M}$ cacodylate buffer solutions (pH 7.1) containing 3\%, $2 \%, 1 \%$, and $0 \% \mathrm{NaCl}$, each step taking $15-20 \mathrm{~min}$. Post-fixation was performed in $1 \% \mathrm{OsO}_{4}$ dissolved in $0.05 \mathrm{M}$ cacodylate buffer, $\mathrm{pH}$ 7.1, overnight at $4^{\circ} \mathrm{C}$. After post-fixation, samples were treated in $1 \%$ tannic acid dissolved in $0.05 \mathrm{M}$ cacodylate buffer ( $\mathrm{pH} 7.1$ ) for $15 \mathrm{~min}$ at room temperature, and then fixed in $1 \% \mathrm{OsO}_{4}$ dissolved in $0.05 \mathrm{M}$ cacodylate buffer ( $\mathrm{pH} 7.1)$ overnight at $4^{\circ} \mathrm{C}$. Samples were dehydrated through a graded series of ethanol solutions, infiltrated with $t$-butyl alcohol and freeze-dried at $4^{\circ} \mathrm{C}$. Samples were coated with platinum-palladium using an ion sputter (E1045; Hitachi Corp., Tokyo, Japan) immediately before observation. We recorded the images using a FESEM (JSM6330F; JEOL Ltd., Tokyo, Japan) at $5 \mathrm{kV}$, and pseudo colored images were generated by Adobe Photoshop. This image shows that mating structure of the female gamete (green) was located on the same side of flagellar beat plane to the eyespot (red), while that of the male gamete (blue) was located in the opposite position, indicating the opposite positioning of the mating structure between female and male gamete in $U$. arasakii.

\section{References}

Miyamura, S., Nagumo, T., Maegawa, M. and Hori, T. 2015. Rearrangement of the flagellar apparatuses and eyespots of isogametes during the fertilization of the marine green alga. Monostroma nitidum (Ulvophyceae, Chlorophyta). Phycol. Res. 63: 284-299.

Miyamura, S., Nomura, M., Mitsuhashi, F. and Nagumo, T. 2018. High-speed video analysis of the flagellar movement of isogametes during fertilization of the marine green macroalga, Chaetomorpha spiralis (Ulvophyceae, Chlorophyta). Cytologia 83: 109-114.

\footnotetext{
*Corresponding author, e-mail: miyamura.shinichi.fw@u.tsukuba. ac.jp

DOI: $10.1508 /$ cytologia. 84.191
} 